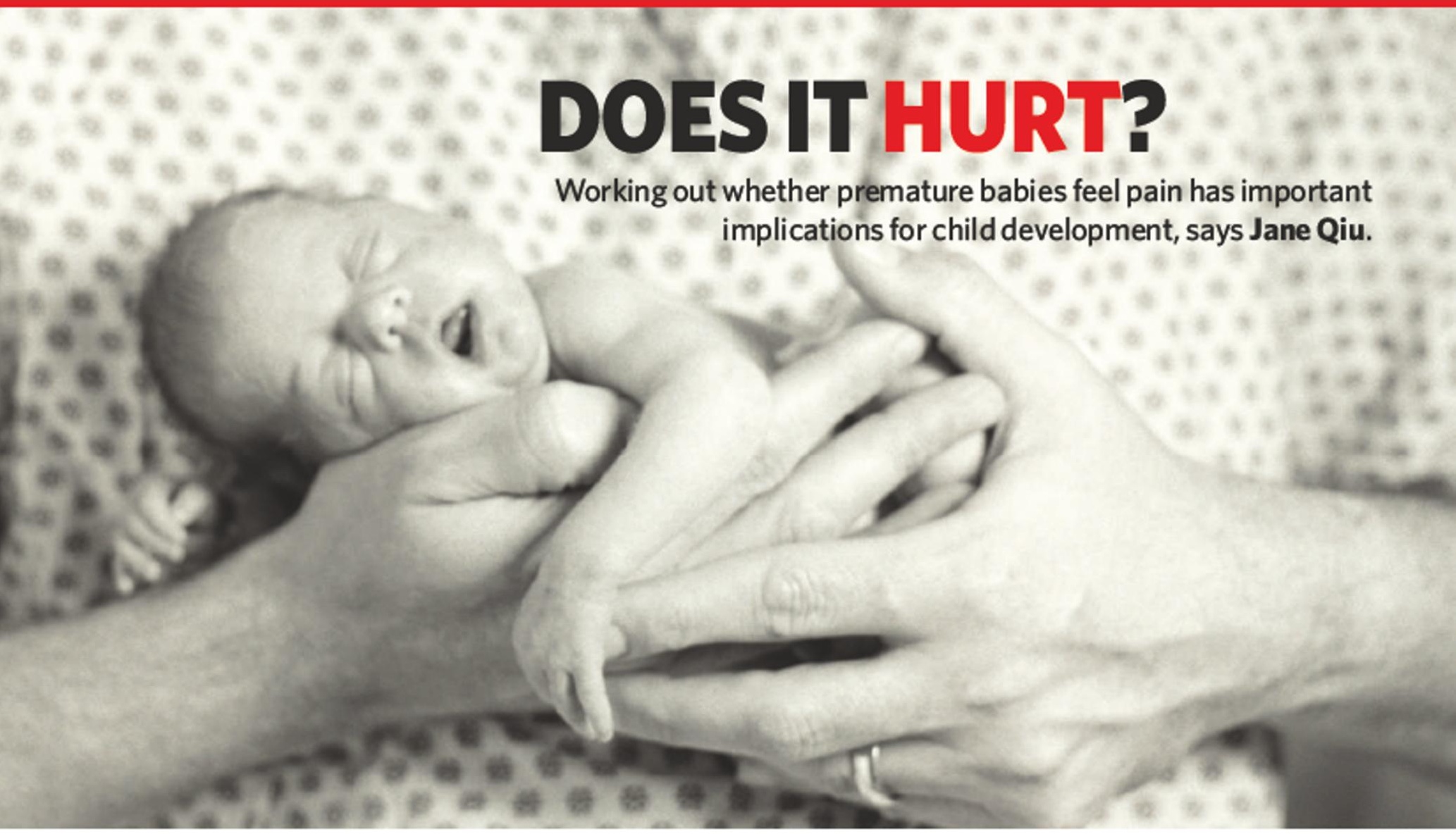

$\mathrm{t}$ was a precarious beginning. Teresa was born 14 weeks earlier than she should have been. Having spent 26 weeks in the womb, she now lies motionless in an incubator in the neonatal intensive-care unit, relying on mechanical ventilation for breathing and intravenous tubes for nutrition. Her tiny fragile body is covered with sensors to monitor blood pressure, heart rate, breathing and temperature.

Teresa's destiny is far from certain. She is suffering from the common complications associated with premature birth, such as breathing difficulties, anaemia and infections. She has to go through many routine medical procedures every day, and also faces a number of major operations in the future.

Teresa is a fictional but representative composite of hundreds of thousands of premature babies born each year. Thanks to medical and technological progress, babies born after 23

"Most intensive-
care units don't have
guidelines. It's all
very patchy and up
to the clinicians and
nurses on duty."
- Judith Meek

use words to explain exactly what they are feeling. For a long time, many health professionals assumed that newborns, and those born prematurely in particular, were too young to perceive pain in the same way that older children and adults do - or if they did, were not able to remember it. They were wary of giving painkillers to infants 'just in case, as the effects and dosing of these drugs had been tested only in adults. Some neonatal clinics started to give painkillers to premature babies during certain medical procedures in the late $1980 \mathrm{~s}$ but, as yet, there is no consensus on best practice.

${ }^{\alpha}$ Most intensive-care units have no guidelines for managing pain in preterm babies, so it's all very patchy and up to the individual clinicians and nurses on duty," says Judith Meek, a paediatric consultant at the Elizabeth Garrett Anderson and Obstetric Hospital in London.

The question of whether weeks' gestation in developed countries are now routinely kept alive. But many of them have lifethreatening complications. It is estimated that each baby in a neonatal intensive-care unit is subjected to an average of 14 procedures a day - a number that can go up to 50 in extreme cases $^{1}$. Although clinicians and nurses intuitively feel that most of these procedures may be painful, there is no easy way to discover whether the babies are feeling anything.

It's a vexed question. Medics rely on selfreporting for pain assessment, but babies can't preterm babies feel pain goes beyond the obvious ethical and humane issues. In recent years, neuroscientists have unearthed evidence suggesting that the nervous systems of premature babies who experience repeated procedures that are potentially painful develop abnormally. Such children may be either excessively susceptible or desensitized to pain as they get older. The problem is likely to increase in the future, as the number of premature births has been rising in westernized countries ${ }^{2}$. This is partly because of the increased number of multiple births resulting from more widespread use of $\vec{s}$ fertility treatments.

There are further implications for neuroscientists too. They hope that, as well as answering the question of whether premature babies feel pain, their results will also help them understand more about how an infant's nervous system develops. This could form the groundwork for the development of much-needed drugs specifically designed for children, whose physiology is often different from that of adults.

\section{Pain barrier}

At the moment, practitioners use 'pain-coding scales' to assess the amount of pain babies might feel. Each scale consists of a set of behavioural criteria, such as crying, withdrawal and facial expressions, as well as physiological indicators, such as heart rate, blood pressure and breathing patterns. "All those measures have their strength," says Maria Fitzgerald, a neuroscientist at University College London. "But they may not be true reflections of pain experience, as they can also change under other stressful conditions, such as hunger and cold."

Part of the problem is that the experience of pain is far more than just a straightforward physiological response to stimuli. Being a psychological state, pain is always subjective, and is coloured by a number of cognitive and emotional factors. It can result from nociception - the physiological detection of noxious stimuli by pain receptors and nerves - but can also occur independently of this, as a sufferer of phantom-limb pain will attest.

Animal studies suggest that nociception is 
likely to occur in premature infants ${ }^{3}$. But to work out whether premature babies experience pain, neuroscientists need to tackle two key questions. The first is whether the signals generated by the stimuli actually reach the cerebral cortex, the part of the brain that is crucial for the perception of bodily sensation. The second is: to what extent are premature infants conscious, that is, sufficiently self-aware to experience and be emotionally distressed by pain? Have they yet developed minds?

The debate over whether pain signals reach the cortex in premature babies was fuelled by work first published in 2002 by Tim Oberlander, a paediatrician at the University of British Columbia in Vancouver, Canada. He showed that premature babies with severe brain injuries that disable much of the cortex have the same facial expressions and behaviours in response to potentially painful stimuli as normal babies of the same age 4 . This suggested that the behavioural and physiological responses of premature babies to such stimuli are merely reflexes put together at the level of the spinal cord and brain stem. If signals of pain do not go beyond the brain stem, it is unlikely that preterm infants can feel pain.

Recently, however, a number of scientists have attempted to see inside the brains of premature babies to find out if this is the case. Two teams of researchers, one led by Fitzgerald, the other by Kanwaljeet Anand at the University of Arkansas for Medical Sciences in Little Rock, have independently shown that the cortex of premature babies is activated in response to potentially painful stimuli $i^{5,6}$. Both groups used near-infrared spectroscopy (NIRS), a non-invasive imaging technique that can measure subtle changes in the concentration of the blood's oxygen-carrying molecule haemoglobin.

Neuronal activity in the cortex is coupled with changes in the blood flow to different regions of

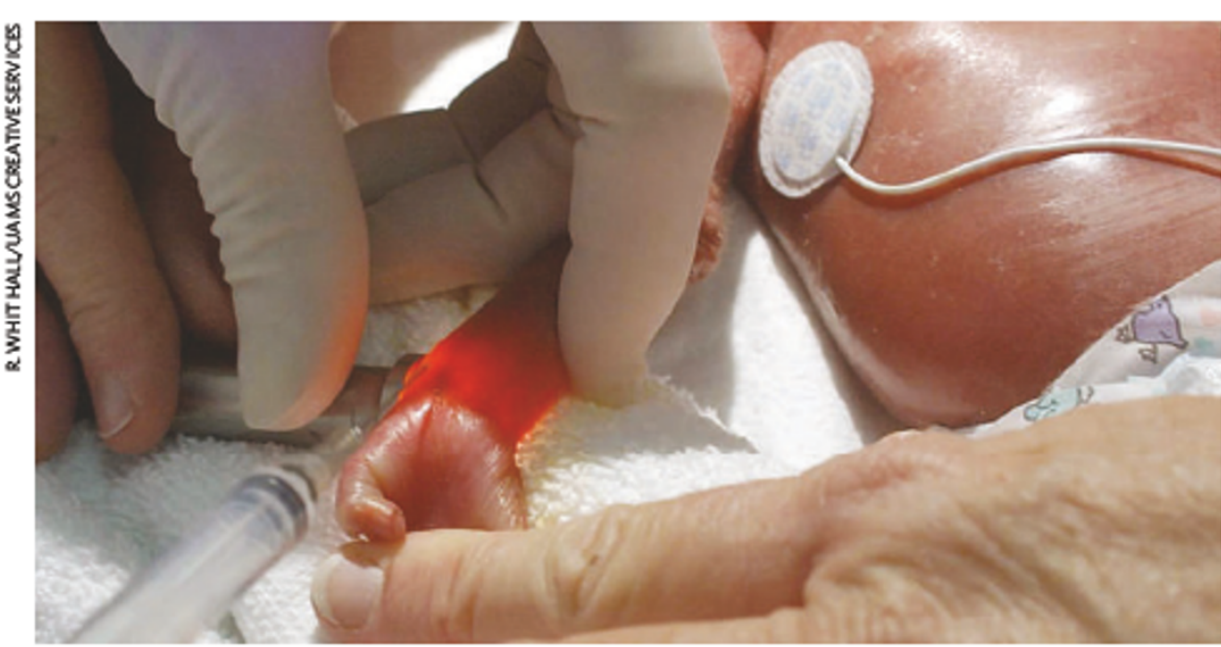

Instant reaction: behavioural responses to pain could be merely reflexes in the nervous system.

the brain, and this is reflected by the ratio of oxygenated haemoglobin to its deoxygenated counterpart. Because of this, NIRS can be used to monitor neuronal activity in the cortex. It is not as sharp or accurate as other imaging systems, such as functional magnetic resonance imaging (AMRI) and positron emission tomography (PET). But these systems would involve moving a very sick and fragile infant into a noisy and stressful environment. NIRS can be performed by simply placing sensors on a baby in its cot.

Using this method, Fitzgerald's team measured cortical activity in response to a heel lance, a widely used procedure for collecting small blood samples, in 18 babies of between 25 and 45 weeks of gestational age, and postnatal ages ranging from 5 to 134 days $^{5}$. Unexpectedly, even in the youngest babies, there was a small but very clear evoked activity in the somatosensory cortex, the part of the cortex that processes bodily sensation.

A brief, non-invasive mechanical stimulation of the heel did not produce detectable cortical activation, even when babies withdrew their feet in reflex action. The researchers found that the older the baby, the bigger the response in the cortex.

\section{Conscious thoughts}

In a parallel study, Anand and his team analysed 40 preterm babies, born at 28 to 36 weeks of gestation, at 25 to 42 hours after birth ${ }^{6}$. They used vein puncture, another way of collecting blood samples, to generate potentially painful stimuli. Following either tactile (skin disinfection) or painful (vein puncture) stimuli, there was an increase in blood flow to the somatosensory cortex, but not to other areas of the cortex. The increase induced by vein puncture was much larger than that by skin disinfection, supporting the findings made by Fitzgerald's team. But unlike Fitzgerald, Anand found that

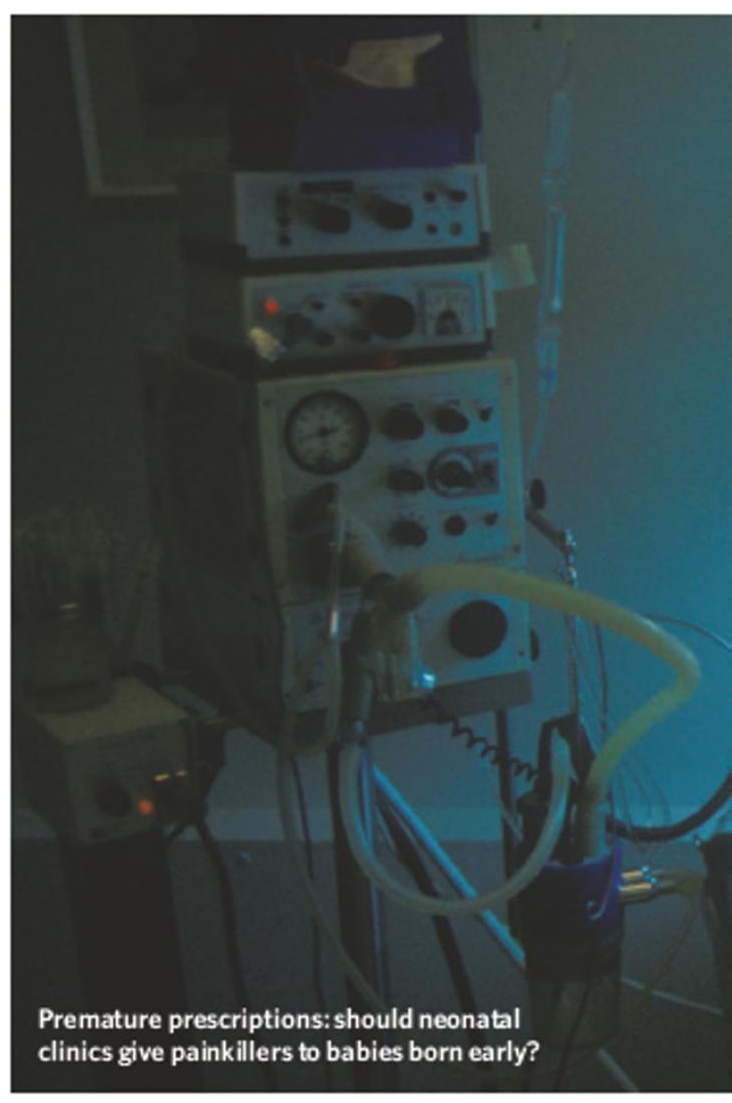

the more premature the baby at birth, the bigger the response to painful stimuli.

This difference between the two studies may be explained by the fact that the teams used different stimuli and babies of different ages. Even so, they do have the same central finding in common: there is activation of the cortex following acute pain in premature babies. The finding is met with great enthusiasm from other pain researchers.

"To make this kind of breakthrough in human neonates is extremely difficult," says Ruth Grunau, a paediatric psychologist also at the University of British Columbia. "It presents a major step forward in the field. But proof of cortical activation in babies is not exactly the same as proof of consciousness.

So how sure are the researchers that those preterm infants do have the same subjective experience that adults recognize as pain? There is simply no proof of consciousness, even in wide-awake adults. And recent studies have raised the question of whether comatose adults, who are also unable to speak, might have some level of consciousness, although this remains highly controversial'. So proving what a baby feels is currently impossible. "How can you take any biochemical or biophysical measures and equate that to a subjective, conscious experience?" asks Anand. "It is a conundrum.

Anand thinks that the best way forward, for now, is to infer that premature babies can feel pain. "If a baby manifests the same behavioural and physiological responses to pain as seen in adults, together with activation of the cortex, then we can be fairly confident that he is feeling pain, even though that's no proof of conscious- 


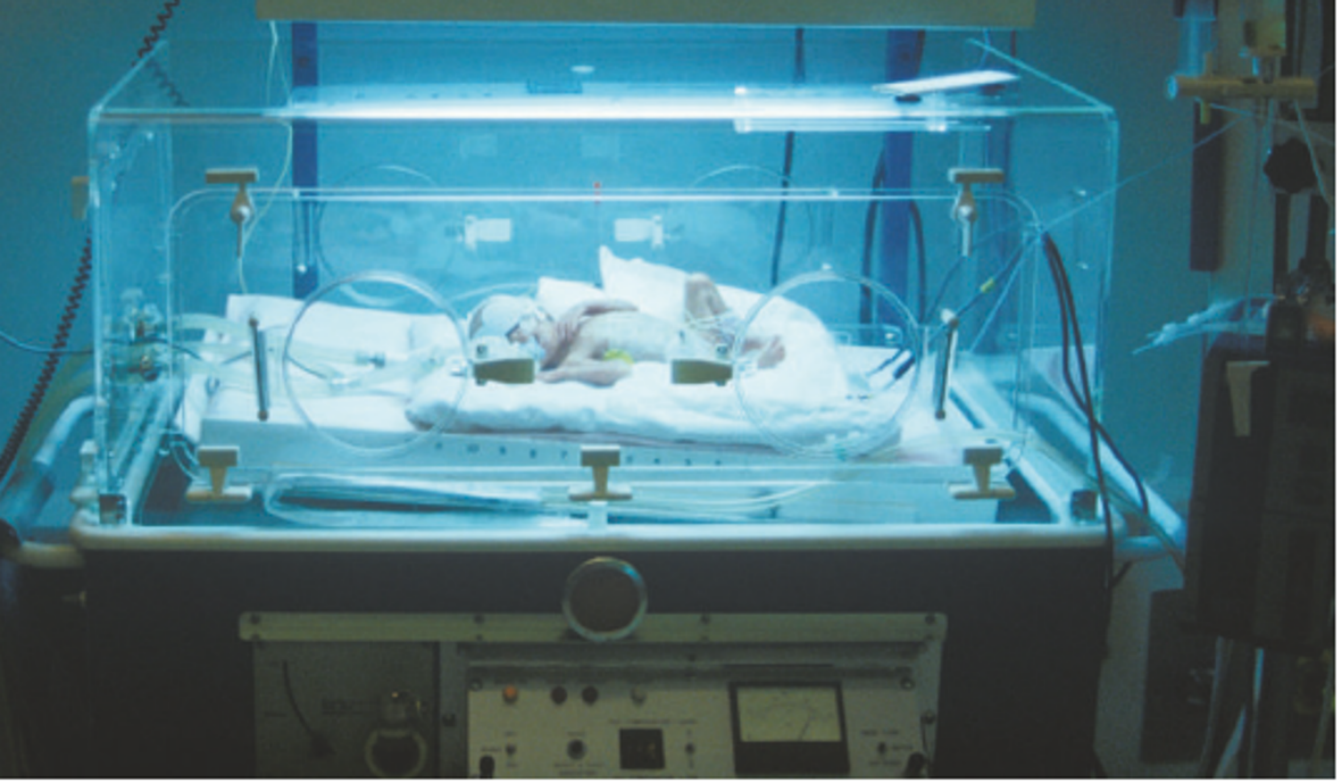

ness," Grunau agrees. "We would seem to be holding an extraordinary standard if we didn't infer pain from all those measures."

That being the case, the issue of alleviating pain and suffering becomes paramount, especially now that scientists know that repeated painful experiences can alter the development of the nervous system. Some studies show that children born prematurely become less sensitive to pain, whereas others demonstrate that children, especially those who were born prematurely and who have had multiple operations early in life, need a higher dose of analgesics when they need further surgery $y^{8}$. In other words, early repetitive pain experiences can either increase or dampen sensitivity to pain later on in life. ${ }^{\alpha}$ There are a lot of confounds here, ${ }^{\infty}$ Fitzgerald acknowledges. This is not surprising, given the complexity of the phenomenon and the difficulties with human neonate research.

\section{Subjective responses}

Studies with newborn rats also show that, depending on the timing, nature and duration of the painful stimuli, the animals become either under-or over-sensitive to pain later on. Both are potentially problematic: children who are less sensitive to pain may be more prone to injury, whereas those who are hypersensitive may withdraw from everyday activities.

Grunau is also concerned about changes in such children's stress responses. She and her colleagues have conducted detailed studies to correlate the amount of potentially painful procedures premature babies undergo in intensive-care units with their levels of stress hormones at various stages of early postnatal development. They found that premature babies of 8 and 18 months have much higher levels of stress hormones than their full-term counterparts $^{9,10}$, although how long these effects persist remains unknown.

A large body of animal and human studies has shown that prolonged exposure to stress hormones can result in changes in the brain, especially in areas important for learning and memory ${ }^{11}$. "Preterm babies are vulnerable because the structure of their brains is still being formed," says Meek. "This is really worrying."

Detailed analysis of a large number of children between the ages of 5 and 14 indicates that children who were born prematurely are about 2.6 times as likely to have attentiondeficit disorder as their fullterm equivalents ${ }^{12}$. Such children also tend to have various learning difficulties, increased levels of anxiety and poorer cognitive outcome. It is difficult to pinpoint the precise causes of these differences - after all, prematurity brings a host of problems that could all contribute in one way or another. But, says Grunau, pain is certainly on this list of potential factors.

So researchers are trying to develop ways to measure pain more accurately in premature babies. Fitzgerald is now testing how well cortical activity in response to potentially painful stimuli correlates with subjective assessment of pain by medical practitioners. She is also using imaging to monitor the efficacy of painkillers in the babies. To refine the findings with NIRS, her team is now using electroencephalography, which will allow the group to map how input from nerves is transmitted and processed in various regions of the preterm baby's cortex. Anand and his colleagues are carrying out similar kinds of studies including the effects of prolonged pain, such as post-operative pain and discomfort from being on a ventilator.

Anand's team is also using fMRI and other imaging techniques to broach the highly controversial and politically sensitive question of whether the fetus can feel pain. Researchers agree that studies on premature infants won't address this question. The process of being born alters an infant's brain physiology so radically that it cannot readily be compared to that of a fetus still in the womb. Even with the help of sophisticated imaging technology, tackling this question will be far from easy.

Studies of pain in premature babies could, however, fuel an initiative to develop painkillers designed especially for babies and children. Drug regulatory bodies, government funding agencies and charities have now realized this is an important issue.

In the United States, the Best Pharmaceuticals for Children Act of 2002 authorizes government agencies, in particular the National Institutes of Health and the Food and Drug Administration, to help develop drugs specifically for children. Task forces of scientists, clinicians and ethicists specialize in various conditions affecting newborns, including pain as well as neurological, cardiovascular and respiratory diseases. The initiatives aim to set priorities for scientific research and to establish ethical guidelines for clinical trials using babies.

One such task force, the Neonatal Pain-Control Group, ${ }^{\alpha}$ is one of the top priorities among all the task forces," says Anand, its chairman. "We expect to see some major clinical advances in neonatal pain management the next few years.

Jane Qiu is a science writer based in London

1. Simons, S. H. P.et a. Arch Pealiat. Adolesc. Med 157, 1058 1064(2003)

2 Kramer, M. S etal. J. Am. Med Assoc. 280, 1849-1854 (1998).

3 Fitzgerald, M. Nature Rex Neurosd. 6, 507-520 (2005)

4. Oberlander, T.F.et al. Pediatrics $110,570-576$ (2002).

5. Slater, R et al. J. Neurosci. 26, 3662-3666 (2006).

6. Bartocoi, M. etal. Pain 122, 109-117 (2006).

7. Hopkin, M. news chature hittp://Www.nature.com/ news/2006/0609 04/full/060904-11 html(2006)

8. Peters, J.W.et al. Pain 114, 444-454 (2005).

9. Grunau, R. V. E etal. Pain 58,341-346(1994)

10. Grunau, R V.Eet al. Sem Fet Neanat.Med 11, 268-275 (2006).

ii. Kloet, E R, Joels, M. \& Holsboer, F. Nature Rev. Neurosci. 6, 463-475 (2005)

12. Bhutta, A. T. etal. J.Am. Med Assoc. 288, 728-737(2002) 